add a second signal processor. Violá! Stereovision. With an appropriately chosen objective lenses, positioned correctly, we now have a $10 \mathrm{X}$ digital stereo Mousescope ${ }^{T M} .20 \mathrm{X}$ and so on are equally doable.

Not being satisfied with this, we are currently working on replacing the single objective with a two element design, with a movable second lens in front of the first lens, acting to zoom the magnification.

Now we can not only zap the bad guys, but take rapid digital micrographs of reasonable quality of any surface on which we place the mouse. Very useful for following live cells, surface reactions and microcorrosion, on-chip micromachines, and just what those bloody dust mites in our pillows are up to anyway.

There is still some fiddling with software, perhaps Photoshop, to integrate the images from the two cameras and processors. These would then be shown on the computer monitor to produce stereo imaging. We admit that this isn't going as well as hoped. Color anaglyph images interfere with the hoped-for color imaging, and polarized images with polarized viewers don't cooperate well with LCD monitors.

But as soon as we link this issue to bioterrorism, we'll be able to get a multi-million dollar DARPA grant, hire a battery of programmers and monitor engineers, and solve the problem in no time. What's a few million dollars for software and monitor design, when with a few simple modifications of the ubiquitous computer mouse we have invented a cordless, go-anywhere $\$ 30$ digital stereomicroscope? $\odot$

Lee van Hook

Piltdown Research Institute, Münchhausen University

\section{A Quick Method for Safely Restraining Mouse Pups for Microscopy}

I visualize green-fluorescent protein labeled mouse pups with a Leica stereoscope setup (not using confocal). On the stereo scope I often look at newborn pups or pups that are a few days old. I have used a piece of plastic wrap to hold them still (not too tight) and it holds the skin taut which helps. To do this without harm to the pups, I have been taping one edge of the plastic down and then holding the other side with my free hand. Light tension is all that is needed, and it never seemed to bother even one day old pups. By using tension the actual pressure is quite light. The pups seem to naturally stop wiggling with this pressure (like having mom sit on you in the nest?) and the light from the scope. To keep the pups warm we got a 37 degree heated mat from Harvard which warms the whole stage when placed underneath.

\section{Michael J. Herron, University of Minnesota herro001@umn.edu}

\section{A Simple Image Archive That's Cheap, Too!}

Our group has many archived Kodachromes, EM micrographs and digital images, and continously produces new images in all formats. Primarily, these are light micrographs of hematoxylin \& eosin stained tissue sections and immunohistochemistry slides, but they also include electron micrographs and confocal micrographs. It has been a continous problem to keep track of all these images, since

\section{IT'S SHOW TIME!}

Watch Your SEM Perform with Quality Scintillator Light Guides From

\section{E. Taylor Engineering, Inc.}

- Light Guides for Leo/Cambridge model SEMs - $\$ 495$

- Custom Scintillators for other model SEMs - $\$ 89$

- P47 Phosphor Coating

- Highest Quality Engineering

- See our web site for special offers on maintenance and re-coating specials

M. E. Taylor Engineering, Inc. 21604 Gentry Lane Brookeville, MD 20833 Phone: (301) 774-6246

See us on the Web@ www.semsupplies.com
"The Evactron device can significanty reduce contamination in the SEM."

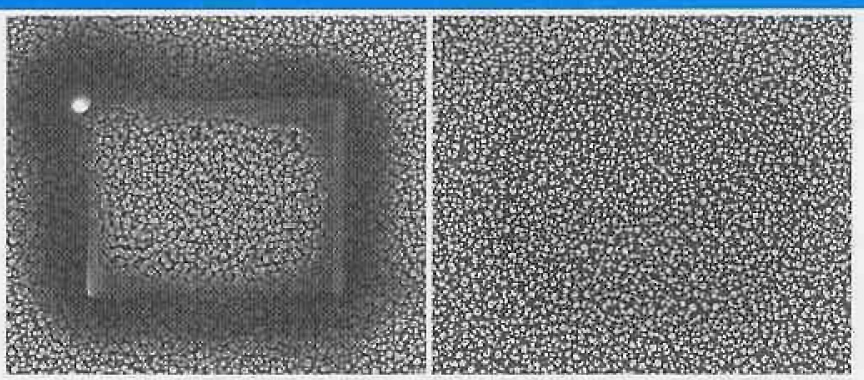

A silicon "grass" sample irradiated for 10 minutes before (left) and after (right) the use of Evactron SEM-CLEAN device. $50 \mathrm{kX}$ From Active Monitoring and Control of Electron Beam Induced Contamination by Andras E. Vladar, Michael T. Postek and Ronald Vane" "Active Monitoring and Control of Electron Beam Induced Contamination" Proc. SPIE Vol. 4344 (2001), 835

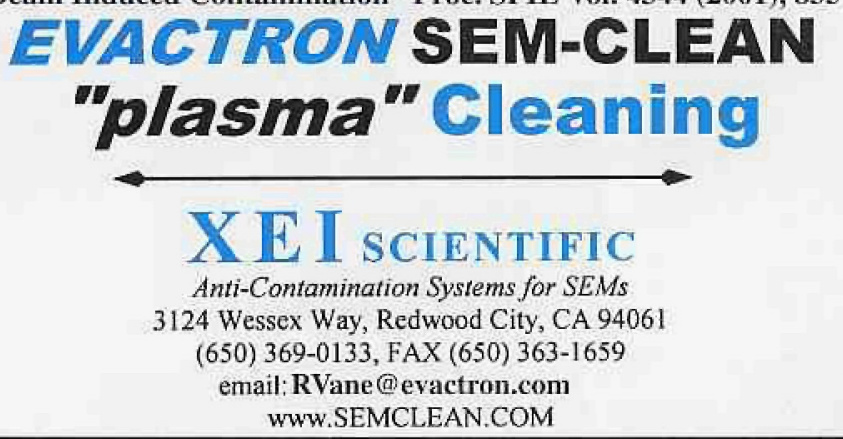




\section{IIIOROSoopy 101}

every researcher tends to keep their own binders and electronic files, making it difficult to know exactly what we have available. We have a central lab database built with Microsoft Access, which is accessible by everyone in the group from their desktop computers. Our goal was to have an image archiving system that would allow anyone in the group to see what we have in our extensive files using the database to search by any criteria. We had built a system in our database that satisfied most of what we wanted as far as cataloging and searching. The big problems were: 1) entering the data, especially for archived images, 2) producing thumbnails for view in the database (very time consuming), and 3) scanning and archiving the images at publishable quality on the servers. This last requirement was especially onerous since the LAN people do not want the servers filled up with images.

We found a solution in two products. First, we aquired a PowerFile CD/DVD changer that accomodates 200 disks and has two drives ( $\$ 1,800$, www.dvdchanger.com). This gives us "near-line" storage for images that are not used very often, but that are available when wanted and within a reasonable time ( 20 to 30 seconds). The Powerfile is connected to a local machine via Firewire and then shared over the network. A server version is also available. With 200 DVD disks, the Powerfile can hold up to $900 \mathrm{~GB}$ of data. Ten Powerfiles can be daisy chained if more storage is required.
Secondly, we use Thumbs Plus ( $\$ 350 / 5$ users, www.cerious.com) as the cataloging software. Thumbs Plus will automatically catalog any file or drive, either local or network, and produce thumbnails. Within Thumbs Plus we can add user-defined fields to hold our unique identifiers for each image. ThumbsPlus uses the MS-Access JET database engine that makes it a very simple matter to link to any other Access database. Additionally, it will connect by $O D B C$ protocols to many other database programs. These features made it ideal for our purposes. Presently Thumbs Plus is designed to be the "parent" database. Cerious Software is working on allowing it to be the data source, however. The way we use it is to put a button on an Access form and open it from Access to search for images. With the links from Thumbs Plus back to our Access database we can use any field to perform searches.

Within our database we can now log images as we take them. The images are then burned on CD's or DVD's and put in the PowerFile. We then use ThumbsPlus to catalog those images and produce thumbnails. We add a unique identifier that links back to our main Access database, thereby linking to all information from a given case. With this setup anyone can now access any image that has been put into the PowerFile without leaving their work area.

Tim Morken

Centers for Disease Control and Prevention, Atlanta email tim9@cdc.gov

Scalpels to Scoops to Screwdrivers to Spatulas to Speedles to Sputter Coaters Carbon Coaters to Clip Mounts to Carbon Rods to Custom Equipment Beakers to Beam Stops to Boats to Books to Bottles to Boxes Tape to Timers to Titanium Tweezers to Tensile Testers Pipettes to Planchets to Pithwood to Power Supplies Hacksaws to Hex Grids to Heating Stages Universal Holders to Uranyl Acetate Vacuum Pumps to Viewing Boxes Wafer Tweezers to Work Holders Magnifiers to Micromanipulators Desiccators to Dropping Bottles Latex Spheres to Lens Tissue EFFA Dusters to Evaporators Glass Bottles to Grid Boxes Razor Blades to Ruby Mica Ferritin to Films to Forceps Acetone to Apertures ERNEST F. FULLAM, INC.
900Albany Shaker Road
Latham NY 12110-1491 What can you imagine?

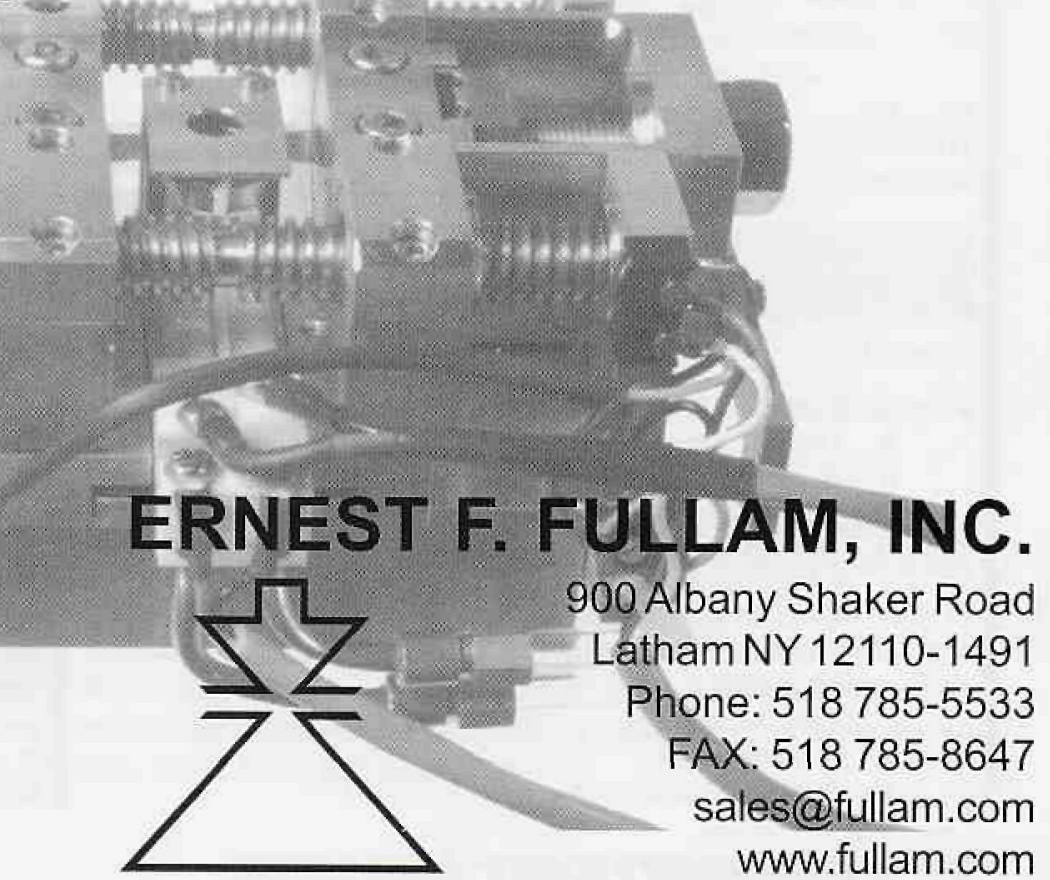

